\title{
Maternal and obstetric risk factors for low birth weight and preterm birth in rural Gambia: a hospital-based study of 1579 deliveries
}

\author{
Abdou Jammeh ${ }^{1,2^{*}}$, Johanne Sundby ${ }^{1}$, Siri Vangen ${ }^{3}$

\begin{abstract}
${ }^{1}$ Section for International Health, Department of General Practice and Community Medicine, Institute of Health and Society, University of Oslo, Oslo, Norway;

${ }^{2}$ Reproductive and Child Health Programme, Ministry of Health and Social Welfare, Banjul, Gambia;

${ }^{3}$ Oslo University Hospital, Department of Obstetrics and Gynaecology, National Resource Centre for Women's Health, Oslo, Norway. E-mail: ${ }^{\text {abdoujammeh777@,hotmail.com, abdou.jammeh@studmed.uio.no }}$
\end{abstract}

Received 2 June 2011; revised 25 August 2011; accepted 4 September 2011.

\begin{abstract}
Introduction: Low birth weight and prematurity are risk factors for perinatal morbidity and mortality, which is high in Sub Saharan African countries. We determined the frequency of and maternal and obstetric risk factors for low birth weight and preterm birth among hospital births in rural Gambia. Method: We performed a hospital-based retrospective analysis of deliveries from July to December 2008 in two rural hospitals. Maternity records were reviewed and abstracted of the mother's demographic and reproductive characteristics, obstetric complications and foetal outcome. The maternity records contain important information maternal health and complications during pregnancy and intrapartum period. The records also contain information about the newborn's vital status and birth weight. To determine the association between low birth weight (LBW), preterm birth (PTB) and maternal demographic characteristics and obstetric complications we calculated odds using logistic regression. Main outcome measure(s): Low birth weight ( $<2500$ grams) and preterm birth ( $<37$ weeks). Results: Our final sample included 1244 singleton live births with complete information about all variables. The rate of $L B W$ and $P T B$ were $10.5 \%$ and $10.9 \%$ respectively. Ninety-four percent of LBW infants were estimated to be preterm births. The mean birth weight was $3013 \mathrm{~g}$ (541 g standard deviation-SD), while the mean gestational age was 37 weeks. The pattern of risk factors was similar for LBW and PTB and both were strongly associated with antepartum haemorrhage and hypertensive pregnancy disorders. Additionally, primi parity was a risk factor for both PTB and LBW. Conclusion: The percentage of low birth weight and preterm birth in rural hospitals in
\end{abstract}

The Gambia is high. The most significant risk factors were those that may be detected during the antepartum period. Thus, vigilant monitoring during pregnancy, early detection and management of obstetric complications coupled with provision of timely obstetric care interventions are crucial for reducing

Keywords: Adverse Birth Outcomes; Low Birth Weight; Preterm; the Gambia

\section{INTRODUCTION}

Low birth weight (LBW) and preterm birth (PTB) represent major public health problems in developing countries, and are major determinants of perinatal survival as well as infant morbidity and mortality [1,2]. Data on LBW rate tend to be quite diverged. Every year it is estimated that 18 million LBW babies are born globally, making up nearly $16 \%$ of all live births [3]. More than $95 \%$ of the low birth weight babies are born in developing countries. The estimated level of LBW in developing countries $(16.5 \%)$ is two-fold higher than the level observed in developed countries (7\%) [4]. Of the 20 million LBW infants born in 2005, more than half were born in South Asia; representing a low birth weight rate of $29 \%$ [5]. In sub-Saharan Africa the incidence of LBW was estimated around $13 \%$ to $15 \%$ with slight variations across the region [4]. In the Gambia, approximately $20 \%$ of infants were estimated to weigh less than $2500 \mathrm{~g}$ at birth. There is slight variation between the urban and rural ar- eas representing $19.7 \%$ and $20 \%$ LBW respectively. However, only $52 \%$ of the total births were estimated to be weighed at birth [6].

Weight at birth is a good indicator for the newborn's chances of survival, growth, long-term health and psychological development [4]. In developing countries 
LBW has been shown to stem from both intrauterine growth restriction and preterm births, while in developed countries it is mainly attributable to preterm birth [7]. Poor maternal health and diseases such as vaginal bacterial infections, pre-eclampsia, malnutrition plus syphilis/HIV and malaria, that have not been adequately treated before or during pregnancy contribute to intrapartum deaths; as well as preterm birth and low birth weight babies [4]. In sub-Saharan Africa, endemic maternal malaria infection, particularly if manifested as placental parasitaemia is implicated to predispose towards low birth weight and preterm delivery [8]. Most at risk of developing malaria are the primigravid mothers as they have not yet acquired the selective immunity, which develops during subsequent pregnancies [9].

The frequency of LBW is an indicator of the risk of perinatal death as well as the populations' health [10]. Hence, the shorter the gestational age, and the smaller the baby, the higher the risk of death and disability [4]. Low birth weight babies are at a greater risk of neurological disorders, such as seizures, cerebral palsy, severe mental retardation, hearing and visual impairment [11]. Thus, data on the frequency and risk factors of LBW and PTB are crucial for the design of maternal and child health programmes, particularly in developing countries [12]. Here many babies are born at home without a skilled birth attendant. These babies are seldom assessed or weighed [13]. The epidemiology of LBW has been extensively explored in developed countries, but in developing countries absence of reliable data on LBW remains a concern even in health institutions. Thus, the aim of this study was to determine the proportion, (distribution) and (obstetric) risk factors for LBW and PTB among hospital births in rural Gambia.

\section{MATERIALS AND METHODS}

\subsection{Study Setting and Design}

This cross-sectional retrospective study was carried out at the rural hospitals called Armed Forces Provisional Ruling Council (AFPRC) Hospital and Bansang General Hospital in the Gambia. These hospitals are located in two different rural health regions, the North and South bank of the Gambia respectively. Together, they serve a population of nearly 600,000. Comprehensive Emergency Obstetric Care (CEmOC) is available most of the time, mainly provided by Cuban Medical Doctors. The two hospitals are referral points for almost 30 peripheral health centres where Basic Emergency Obstetric Care (EOC) is virtually none-existing, thus women with obstetric complications from the North and South Bank respectively are referred to AFPRC and Bansang Hospitals. Many of these women are referred during labour.
In its drive to make health care more accessible to rural populace, particularly women and children, the Government of The Gambia adopted the primary health care (PHC) strategy. Villages with more than 400 inhabitants have resident traditional birth attendants and village health workers who were selected by the communities themselves. The traditional birth attendants have had eight weeks formal training in antenatal, intrapartum and postnatal care of the mother and baby. The emphasis was basically on identification of danger signs during pregnancy, labour and the postpartum period, as well as on clean delivery and timely referrals. Antenatal care (ANC) is by trained health workers, and partly at stationary clinics, (partly) delivered in mobile outreach points. Coverage for one ANC visit is high, about $99 \%$ while institutional deliveries are low (55\%) [6].

\subsection{Study Population}

Information on all births that occurred from $1^{\text {st }}$ July 2008 through $31^{\text {st }}$ December 2008 at AFPRC and Bansang Hospital were extracted from maternity case notes, admission and delivery registers. These standardized delivery $\log$ s and case notes contain vital information about maternal health, complications during antepartum and intrapartum period as well as the newborn baby including birth weight. Doctors or midwives filled these forms upon admission and immediately after delivery. Data extraction was done by the principal investigator and research assistants who were trained nurse/midwives. A pre-coded case abstraction inventory was used.

\subsection{Variables}

The main outcome measures were LBW and PTB rate. We defined LBW as a birth weight of less than $2500 \mathrm{~g}$ irrespective of gestational age. Preterm birth was defined as childbirth occurring at less than 37 completed weeks of gestation. Eligibility criteria was based on the recommendation of the International Classification of Diseases (ICD-10) for international comparison of viability; that is birth weight of $\geq 1000 \mathrm{~g}$ and/or born at $\geq 28$ weeks of gestation [14]. Gestational age was estimated by the number of days between the first day of the last menstrual period (LMP) and date of birth expressed in completed weeks after LMP as recorded in the maternity delivery log. This information is usually entered during the ANC visits, but may be inaccurate for some, especially illiterate women.

For each birth we extracted the following information: demographic and reproductive characteristics such as maternal age in years; three groups $13-19,20-29, \geq 30$ years. Parity categorized as primiparous $0,1-4$ previous deliveries, $\geq 5$ previous deliveries, and residence, as Primary Health Care (PHC) village or non-PHC village. Obstetric factors: antenatal care attendance for present 
pregnancy, presence of obstetric complication(s) such as antepartum haemorrhage, preterm premature rupture of membranes (PPROM), hypertensive pregnancy disorders (severe pre-eclampsia/eclampsia) with a minimum diastolic blood pressure $\geq 110 \mathrm{mmHg}$ and proteinuria $(++)$. This lower limit for diastolic blood pressure is consistent with what is reflected in the national guideline [15]. Foetal characteristics included sex, birth weight and gestational age at birth, plus vital outcome.

A total of 1849 maternity admissions were recorded during the six months period. We excluded from the analysis 224 women (12.1\%) who had not delivered, 21 births without information on the vital status, birth weight and/or gestational age, 25 infants that weighed less than $1000 \mathrm{~g}$ and 240 stillbirths. Also excluded from the analysis were 95 sets of live born twins as they represent a special high risk group for LBW and PTB. The final data set comprised of 1244 singleton live births in which birth weight was available (Figure 1). This study was approved by the Ethics Committee of Norway and the Joint Gambia Government and Medical Research Council Review board. Permission to carry out the study was accorded by the chief executive officers of the two hospitals and the Ministry of Health of The Gambia.

\subsection{Statistical Analysis}

We executed a descriptive analysis of the results through frequency analysis and cross-tabulation to determine the percentage of LBW and PTB calculated as a proportion of all singleton live births. We compared the mean birth weight of complicated and uncomplicated deliveries using the t-test. To establish the relationship between LBW/PTB and the risk factors, a chi-square test was applied or Fisher's exact test where appropriate. The odds ratio (OR) and corresponding 95\% confidence intervals (CIs) of the risk factors were estimated using a multivariate logistic regression. All p-values were two sided and values of 0.05 were regarded statistically significant. Statistical analysis was executed with Software Package for Social Sciences (SPSS) for Windows, version 16.0 (SPSS Inc. Chicago, IL, USA).

\section{RESULT}

A total of 1579 pregnant women delivered at two Gambian rural hospitals during the second half of 2008 of which 1244 (78.8\%) were singleton live births. Of the recorded live births, the rate of LBW (irrespective of gestational age) and PTB respectively were $10.5 \%$ and $10.9 \%$. Of the total births, the mean birth weight was $3013 \mathrm{~g}$ (541g standard deviation-SD). The mean birth weight among those with obstetric complications was $2951 \mathrm{~g}$ (SD $659 \mathrm{~g}$ ), and $3023 \mathrm{~g}$ (SD $518 \mathrm{~g}$ ) in uncomplicated cases; (95\% CI for mean: 2855.3 - 3047.5 and 2991.9 - 3054.3) respectively. Of the 1579 recorded total births, $240(15.2 \%)$ were stillbirths. The stillbirth rate among LBW infants was $40.5 \%$, compared to $9.7 \%$ in babies with birth weight $\geq 2500 \mathrm{~g}$. Additionally, $94 \%$ of LBW infants were estimated to be preterm births. The

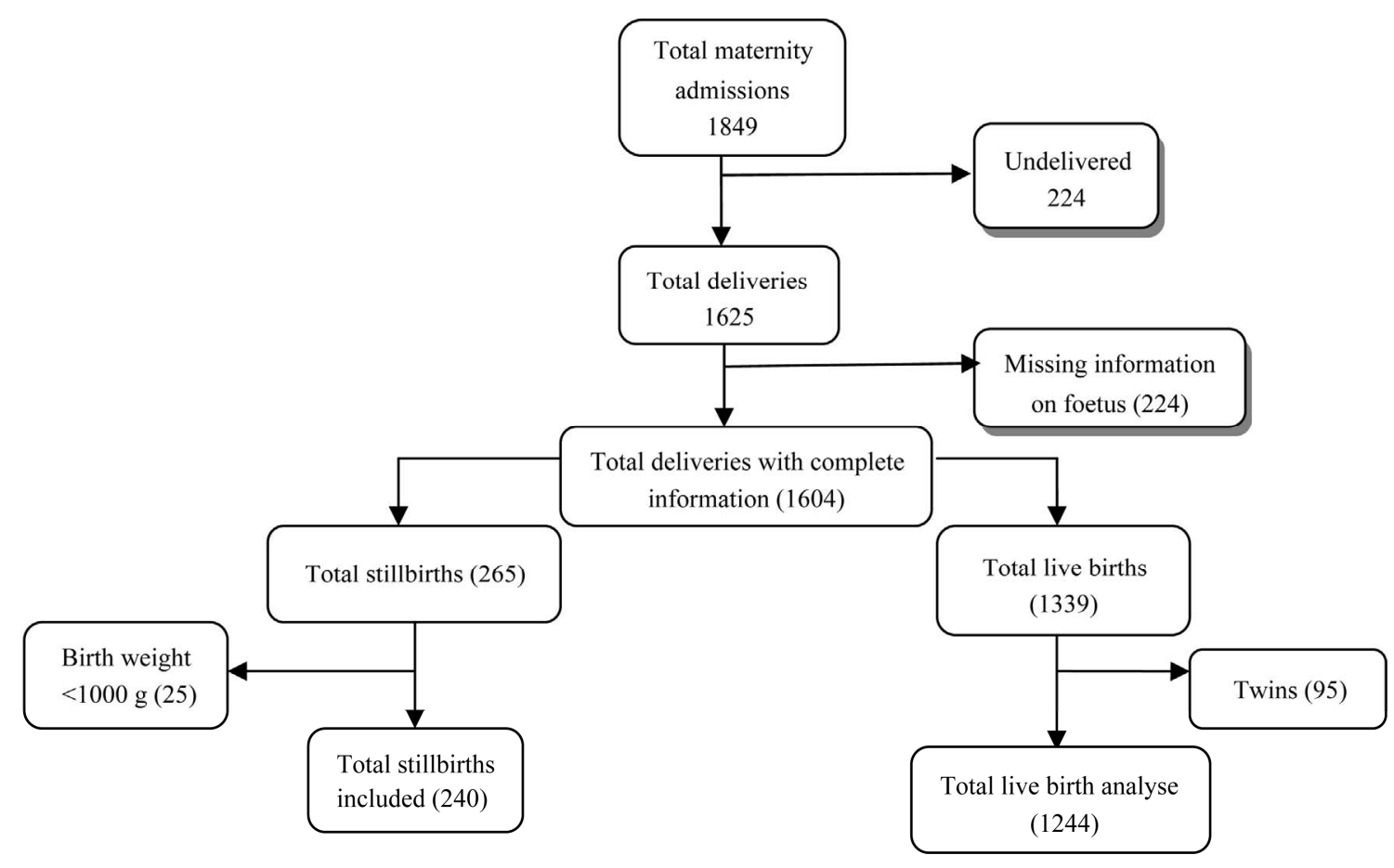

Figure 1. Flow diagram of study population. 
rate of LBW among boys was 9.2\%, compared with $12.0 \%$ in girls; with a mean birth weight difference of $116 \mathrm{~g}(95 \%$ CI for mean 3023.9 - 3075.3 and 1903.1 2992.6) respectively (Table $\mathbf{1}$ ).

Table 2 shows the distribution of the sample according to demographic and obstetric factors. The age of mother ranged from 13 to 48 years, and more than one third was below 20 years of age. About two thirds of the mothers were multiparous and most of them $(72 \%)$ reside in nonPHC villages. There were more male births than females. Nearly all the mothers (99.5\%) had attended antenatal care at least once.

Table 1. Low birth weight rate and mean birth weight among 1244 singleton live born babies according to presence of absence of obstetric complication, sex of baby and gestational age at birth Bansang and AFPRC referral hospitals from July 2008 to December 2008 .

\begin{tabular}{|c|c|c|c|c|c|}
\hline (\%) & Total Births & Live Birhts & $\begin{array}{c}B W T<2500 g \\
\text { n; } \%\end{array}$ & BWTg - Mean (SD & 95\% CI for Mean \\
\hline All & 1579 & 1244,100 & 10.5 & $3012.6(541.1)$ & $2982.5-3042.7$ \\
\hline \multicolumn{6}{|l|}{ Sex of baby } \\
\hline Female & 707 & $696 ; 44.1$ & 12.0 & $2947.9(533.4)$ & $1903.1-2992.6$ \\
\hline Male & 872 & $696 ; 55.9$ & 9.2 & $3063.6(542.0)$ & $3023.9-3075.3$ \\
\hline \multicolumn{6}{|c|}{ Obstetric complication } \\
\hline No & 1201 & $1061 ; 86.7$ & 9.0 & $3023.1(517.7)$ & $2991.9-3054.3$ \\
\hline \multicolumn{6}{|c|}{ Gestational age at birth (Weeks) } \\
\hline$<37$ & 281 & $135 ; 11.0$ & 95.6 & $2053.9(396.1)$ & $1989.4-2121.3$ \\
\hline$\geq 37$ & 1298 & $1109 ; 90.6$ & 0.1 & $3129.3(428.7)$ & $3104.0-3154.6$ \\
\hline
\end{tabular}

BWT-Birth Weight; SD-Standard Deviation; CI-Confidemce Interval.

Table 2. Distribution of study sample according to risk factors analysed among 1244 singleton live births at Bansang and AFPRC referral hospitals from July 2008 to December 2008.

\begin{tabular}{|c|c|c|}
\hline Variable & Live births & Percentage \\
\hline Total & 1244 & 100 \\
\hline \multicolumn{3}{|l|}{ Maternal age (yrs) } \\
\hline$<20$ & 448 & 36.0 \\
\hline $20-29$ & 483 & 38.8 \\
\hline$\geq 30$ & 313 & 25.4 \\
\hline \multicolumn{3}{|l|}{ Parity } \\
\hline 0 & 408 & 32.8 \\
\hline $1-4$ & 620 & 49.8 \\
\hline$\geq 5$ & 216 & 17.4 \\
\hline \multicolumn{3}{|l|}{ Residence } \\
\hline Primary Health Care Village & 341 & 27.4 \\
\hline Non-Primary Health Care Village & 903 & 72.6 \\
\hline \multicolumn{3}{|l|}{ Sex of baby } \\
\hline Female & 548 & 44.1 \\
\hline Male & 696 & 55.9 \\
\hline \multicolumn{3}{|l|}{ Antepartum Haemorrhage } \\
\hline Yes & 11 & 0.9 \\
\hline No & 1233 & 99.1 \\
\hline \multicolumn{3}{|l|}{ Hypertensive pregnancy disorders } \\
\hline Yes & 33 & 2.7 \\
\hline No & 1211 & 97.3 \\
\hline \multicolumn{3}{|l|}{ Pre-term premature rupture of membranes } \\
\hline Yes & 45 & 3.6 \\
\hline No & 1199 & 96.4 \\
\hline \multicolumn{3}{|l|}{ Antenatal care attended } \\
\hline No & 6 & 0.5 \\
\hline Yes & 1238 & 99.5 \\
\hline
\end{tabular}




\subsection{Low Birth Weight}

We presented the results of the univariate and logistic regression analysis in Table 3. From the univariate analysis, younger mothers $<20$ years and primiparous mothers respectively, were 1.8 and 2.5 times more likely to deliver a LBW baby than older mothers who were $\geq 30$ years old and the multiparous with $\geq 5$ deliveries. Obstetric complications also had a strong influence on birth weight. Antepartum haemorrhage and hypertensive pregnancy disorders were highly significantly associated with LBW; (Crude ORs $=5.02$ and 2.86) respectively. Additionally, babies who weighed $<2500 \mathrm{~g}$ were 8.6 times more likely to result in an early hospital neonatal death than those babies weighing $\geq 2500 \mathrm{~g}$ (data not shown). After adjustment for the effect of the significant variables in a multivariate logistic regression model, antepartum haemorrhage was the strongest predictor for LBW (AOR
6.6). Other independent risk factors included hypertensive pregnancy disorders with close to three times (AOR 2.7) increased risk of low birth weight. In addition, primigravid mothers were almost 2.5 times more likely to deliver a LBW baby than multigravid mothers with $\geq 5$ deliveries. Young maternal age $(<20$ years $)$ and preterm premature ruptures of membranes also seemed to be associated with LBW; however, they lost their statistical significance after the multivariate analysis.

\subsection{Preterm Birth}

The results from the univariate and logistic regression analysis of preterm birth are presented in Table 4 . The pattern of risk factors for PTB was similar to that of LBW. The univariate analysis showed that antepartum haemorrhage, hypertensive pregnancy disorders, parity (0 and $1-4)$, preterm premature rupture of membranes

Table 3. Odds ratio for low birth weight according to demographic and obstetric variables among 1244 singleton live born infants at Bansang and AFPRC referral hospitals from July 2008 to December 2008.

\begin{tabular}{|c|c|c|}
\hline Variable & Crude OR (95\% CI) & Adjusted OR $(95 \% \text { CI })^{\mathrm{aa}}$ \\
\hline \multicolumn{3}{|l|}{ Maternal age (yrs) } \\
\hline$<20$ & $1.77(1.09-2.87)^{*}$ & $1.45(0.88-2.37)$ \\
\hline $20-29$ & $1.68(1.11-2.55)^{*}$ & $1.08(0.55-2.13)$ \\
\hline$\geq 30$ & 1 & 1 \\
\hline \multicolumn{3}{|l|}{ Parity } \\
\hline 0 & $2.48(1.33-4.65)^{* * *}$ & $2.48(1.05-5.83)^{* *}$ \\
\hline $1-4$ & $1.46(0.99-2.15)^{* *}$ & $1.21(0.74-1.96)$ \\
\hline$\geq 5$ & 1 & 1 \\
\hline \multicolumn{3}{|l|}{ Residence } \\
\hline Primary Health Care Village & $1.52(1.04-2.23)^{* *}$ & $1.48(1.00-2.19)^{* *}$ \\
\hline Non-Primary Health Care Village & 1 & 1 \\
\hline \multicolumn{3}{|l|}{ Sex of baby } \\
\hline Female & $1.35(0.94-1.95)$ & $0.74(0.51-1.07)$ \\
\hline Male & 1 & 1 \\
\hline \multicolumn{3}{|l|}{ Antepartum Haemorrhage } \\
\hline Yes & $5.02(1.45-17.39)^{* * *}$ & $6.59(1.86-23.36)^{* * *}$ \\
\hline No & 1 & 1 \\
\hline \multicolumn{3}{|l|}{ Hypertensive pregnancy disorders } \\
\hline Yes & $2.86(1.26-6.47)^{*}$ & $2.71(1.18-6.23)^{* *}$ \\
\hline No & 1 & 1 \\
\hline \multicolumn{3}{|l|}{ Pre-term premature rupture of membranes } \\
\hline Yes & $1.91(0.87-4.19)$ & $1.76(0.79-3.95)$ \\
\hline No & 1 & 1 \\
\hline \multicolumn{3}{|l|}{ Antenatal care attended } \\
\hline No & $1.72(0.19-14.83)$ & $0.51(0.06-4.55)$ \\
\hline Yes & 1 & 1 \\
\hline
\end{tabular}

${ }^{\text {aa }}$ Adjustment done for all significant variables listed in the table; ${ }^{*}$ P-value $<0.01 ; * *$ P-value $\leq 0.05 ; * * *$ P-value $<0.005$. 
Table 4. Odds ratio for preterm birth according to demographic and obstetric variables among 1244 singleton live born infants at Bansang and AFPRC referral hospitals from July 2008 to December 2008.

\begin{tabular}{|c|c|c|}
\hline Variable & Crude OR (95\% CI) & Adjusted OR $(95 \% \text { CI })^{\text {aa }}$ \\
\hline \multicolumn{3}{|l|}{ Maternal age (yrs) } \\
\hline$<20$ & $1.99(1.23-3.23)^{*}$ & $1.49(0.92-2.41)$ \\
\hline $20-29$ & $1.74(1.50-2.59)^{*}$ & $1.19(0.61-2.32)$ \\
\hline$\geq 30$ & 1 & 1 \\
\hline \multicolumn{3}{|l|}{ Parity } \\
\hline 0 & $2.87(1.51-5.47)^{* * *}$ & $2.67(1.13-6.34)^{* *}$ \\
\hline $1-4$ & $1.47(1.01-2.14)^{* *}$ & $1.18(0.73-1.89)$ \\
\hline$\geq 5$ & 1 & 1 \\
\hline \multicolumn{3}{|l|}{ Residence } \\
\hline Primary Health Care Village & $1.52(1.05-2.24)^{* *}$ & $1.49(1.01-2.20)^{* *}$ \\
\hline Non-Primary Health Care Village & 1 & 1 \\
\hline \multicolumn{3}{|l|}{ Sex of baby } \\
\hline Female & $1.29(0.90-1.84)$ & $1.29(0.90-1.85)$ \\
\hline Male & 1 & 1 \\
\hline \multicolumn{3}{|l|}{ Antepartum Haemorrhage } \\
\hline Yes & $4.81(1.39-16.64)^{* *}$ & $6.59(1.86-23.36)^{* * *}$ \\
\hline No & 1 & 1 \\
\hline \multicolumn{3}{|l|}{ Hypertensive pregnancy disorders } \\
\hline Yes & $2.73(1.21-6.18)^{* *}$ & $2.58(1.12-5.95)^{* *}$ \\
\hline No & 1 & 1 \\
\hline \multicolumn{3}{|l|}{ Pre-term premature rupture of membranes } \\
\hline Yes & $2.13(1.00-4.52)^{* *}$ & $1.99(0.92-4.33)$ \\
\hline No & 1 & 1 \\
\hline \multicolumn{3}{|l|}{ Antenatal care attended } \\
\hline No & $1.65(0.19-14.21)$ & $1.90(0.21-16.97)$ \\
\hline Yes & 1 & 1 \\
\hline
\end{tabular}

${ }^{a a}$ Adjustment done for all significant variables listed in the table; *P-value $<0.01 ; * *$ P-value $\leq 0.05 ; * * *$ P-value $<0.005$.

and maternal age ( $<20$ and $20-29$ years) were all associated with preterm birth. In the multivariable analysis, while controlling for a number of significant variables, antepartum haemorrhage presented the strongest independent association with PTB (AOR 6.5). The other independent risk factors are primiparity (AOR 2.7) and hypertensive pregnancy disorders (AOR 2.6). Lack of antenatal care seemed to have an increased risk of giving birth to a preterm baby, but this finding failed to attain statistical significance (AOR 1.9, 95\% CI 0.21-16.97). However, the numbers were too small to draw any definite conclusions about this finding.

\section{DISCUSSION}

\subsection{Main Findings}

Our results suggested that the levels of LBW and PTB are high in this population. The prevalence of LBW $(10.5 \%)$ and PTB (10.9\%) may be slightly underesti- mated, but comparable with rates of $13.3 \%$ LBW and $12.3 \%$ PTB earlier observed in another rural settings in Gambia [16]. The reported percentage of LBW in this study is slightly lower than in a previous report published by UNICEF and WHO [4]. We believe that the low rates in our study could be due to the exclusion of neonates $<28$ weeks gestation and $<1000$ g body weight at birth. There were many similarities in the pattern of risk factors for LBW and PTB in this study. The adjusted odds ratios for both outcomes were increased among women with antepartum haemorrhage, those with hypertensive disorders of pregnancy and primigravid women. The mean birth weight and gestational age respectively, were 3012 g (541 g SD) and 37 weeks. Boys were slightly heavier than girls (116 g mean birth weight difference).

\subsection{Methodological Issues}

Due to the lack of nationwide vital registration system in 
The Gambia, reliance on hospital-based data is inevitable. In hospitals, particularly maternity wards, birth weights are routinely measured and recorded within half an hour after delivery. In contrast, most of the babies that are born in home settings are usually not weighed until they come for their first vaccination. Even where babies are weighed at birth, their weights are seldom measured or recorded accurately as most of them are born without a skilled and literate birth attendant. As a result the true population - based birth weight data are difficult to obtain. We extracted data from an institutional setting with maternity records and the data was entered by skilled attendants. Information about birth weight and PTB was missing for a small number of cases only (21 births). Therefore, this study should serve as an important source of data about LBW and/or PTB and the associated risk factors in The Gambia.

Even if a substantial proportion of women deliver at home, earlier studies have demonstrated that most with complications tend to reach health facilities, thus we might have over-estimated the true LBW and PTB rates, but there is no valid way to calculate this bias. However, hospital data may show higher percentage of LBW, PTB, and morbidity compared to population-based estimates. Birth weight preference for round numbers, such as 2500 g or 37 weeks of gestation could have influenced our estimates of low birth weight. We believe that such misclassifications are most likely non-differential and do not influence the presented rates of LBW or PTB in our population. Concentrating on deliveries in two rural hospitals raised issues of selection bias and our estimates of LBW, PTB and morbidity may not be representative of the entire Gambian population. In addition, excluding neonates $<28$ weeks gestation and $<1000$ g body weight may have also biased our results. As the study was a ret rospective analysis of data from maternity delivery logs, we were unable to measure maternal workload, socioeconomic and nutritional factors, which are important contributors to poor foetal growth in low income settings. Another limitation of this study was our inability to assess risk factors such as ascending bacterial infections (gonorrhoea/bacterial vaginosis) and malaria which have important implications for the health of the mother and the growing foetus. Malaria status of the mothers was not collected because it was unknown for a large number of mothers and there was no information to determine the level of placental malarial parasitaemia at delivery. There is a slight seasonal variation in work load and access to food in the field in the Gambia [16], but infections like malaria occur year round. However, the National Malaria Control programme has done tremendous work in the fight against malaria with special emphasis on pregnant women. Thus, to mitigate the adverse effects of malaria in pregnant women, doses of sulfadoxine-pyrimrthamine (Intermittent Preventative Treatment-IPT) are given to women during the second and third trimesters of pregnancy during antenatal clinic visits. This is complemented by mass distribution of long-lasting insecticide-treated nets (LLITNs), early diagnosis (rapid diagnostic test-RDT) and prompt case management.

\subsection{Low Birth Weight and Preterm Birth}

This study determined the prevalence and obstetric risk factors of LBW and PTB among women who delivered at two rural hospitals in The Gambia. The results suggested that the prevalence is high in our study population; $10.5 \%$ and $10.9 \%$ respectively for LBW and PTB which is similar to findings from an earlier study in another rural setting in the Gambia; $13.3 \%$ and $12.3 \%$ [16]. The LBW rate observed in the current study is also consistent with findings reported in district hospitals in Nigeria and Mali; $11.6 \%$ and $12.4 \%$ respectively [17], as well rates in North-East Brazil 10\% [18]. Our findings differ from data on LBW compiled by United Nations Children's Fund (UNICEF) and World Health Organization (WHO) from West African countries and Africa as a whole[4]. In this report the incidence of LBW was estimated at $14.3 \%$ and $15.4 \%$ respectively, in the African region and West Africa respectively. However, a direct comparison of the LBW estimates in our study with that of WHO may not be possible due to differences in methodology applied; particularly divergence in samples, definitions and instruments used. Another study from Harare Central Hospital in Zimbabwe [12] reported a prevalence of LBW and PTB respectively as $19.9 \%$ and $16.6 \%$, which is also higher than our results. This may in part be as a result of the high HIV prevalence in this setting. Our findings also differ from data on LBW reported from another hospital-based case-controlled study in conjunction with a population survey in India[19]. In this study the prevalence of LBW was estimated at $30 \%$. This difference might be in part explained by the methods applied to determine gestational age. In the current study, the length of gestational age was estimated from maternal fundal height. Although such an approach may give inaccurate findings for women with poorly growing foetuses, it was concluded that it be may be used as a proxy for the length of gestation when the date of the last menstrual period is not known, especially in developing countries [20]. The low levels of LBW and PTB reported in our study as compared to the above could also be partly due to the exclusion of newborns $<28$ weeks gestation and $<1000 \mathrm{~g}$ body weight at birth. Furthermore, our data indicated that boys were slightly heavier than girls, with a mean birth weight difference of $116 \mathrm{~g}$.

Birth weight is an important proxy for viability, espe- 
cially where reliable gestational age determination is not available [21]. LBW and PTB are also considered to be one of the leading causes of perinatal mortality and morbidity. Our study demonstrated a very high proportion of stillbirths and early hospital neonatal deaths among LBW babies. The stillbirth rate among LBW babies was $41 \%$, compared to $9.7 \%$ in babies who weighed $\geq 2500 \mathrm{~g}$.

Complications during pregnancy have long been known to increase the risk of adverse birth outcomes. In the current study, obstetric complications were highly significant risk factors for giving birth to a LBW and preterm baby. Of the obstetric characteristics analysed in relation to the frequency of LBW and PTB, mothers with antenatal haemorrhage and hypertensive disorders in pregnancy were more likely to deliver both LBW and preterm babies. Women with antepartum haemorrhage were found to be at increased risk of giving birth to a LBW baby (AOR 6.59, 95\% CI 1.86 - 23.36). This is consistent with findings from a study done in Northern Tanzania [22] and in Colorado USA [23], where 50\% of all births complicated by abruption placenta ended up as LBW. Similar findings were previously reported elsewhere [24]. The increased risk of PTB with antepartum haemorrhage has also been reported by other researchers [19], which corroborated well with our findings. In addition, bleeding during pregnancy could be due to low implantation of the placenta in the less vascularised lower segment leading to intrauterine nutritional deprivation of the foetus and growth restriction [25]. Thus, bleeding during pregnancy can be used to identify women at risk of having LBW babies and PTB [19].

Specific pathologies such as severe pre-eclampsia are known to increase the rates of PTB and LBW. Hypertensive disorder of pregnancy is considered to be a major worldwide problem and presents an increased risk of both maternal and perinatal mortality and morbidity [26, 27]. Preeclampsia, a multisystem disorder partly of unknown aetiology specific to pregnancy [28], has been implicated as one of the main predisposing factors for intrauterine foetal growth restriction and prematurity[29]. Consistent with findings from studies conducted elsewhere $[19,25,30]$; a hypertensive pregnancy disorder (pre-eclampsia) was also observed to significantly increase the risk of LBW (AORs 2.71; 95\% CI 1.18 - 6.23) and PTB (2.58; 95\% CI 1.12 - 5.95) in the current study. Additionally, in their study, Yücesoy et al. [31] demonstrated that gestational age and neonatal birth weight were lowest in severely pre-eclamptic mothers. This may be as a result of the utero-placental insufficiency that happens in this category of disorders which can result to foetal growth retardation [25]. Our findings reaffirmed the negative contribution of obstetric complications on birth weight and gestational age. Thus, adequate moni- toring and control for obstetric complications during pregnancy may contribute to the attainment of optimal gestational age and intrauterine growth [32]. In addition, the reduction of LBW and PTB could be key catalysts towards the attainment of Millennium Development Goal four (MDG-4) for reducing child mortality[4]. With the target of the MDGs in sight, preeclampsia/eclampsia needs to be recognized as priority areas in reducing maternal mortality [33], as well as prenatal morbidity and mortality in developing countries. Thus, improved access to appropriate obstetric care, and better screening, prompt diagnosis and treatment of antepartum haemorrhage and preeclampsia/eclampsia are warranted to reduce the consequences of these obstetric complications on birth weight and gestational age in developing countries, including The Gambia. As most women (99\%) in The Gambia received ANC at least once during pregnancy [6], this could be an important entry point for the prevention of and identification of basic detectable maternal problems like anaemia, malaria and high blood pressure.

Antenatal care (ANC) is one of the "four pillars" of Safe Motherhood and is practiced all over the world. It provides a critical platform for influencing a woman to select a skilled provider for birth, and to establish a plan for normal birth as well as emergency plan [34]. Even though effective ANC alone may not prevent maternal and newborn mortality globally, the care a woman receives during pregnancy plays a critical function in assuring the healthiest possible outcome for the mother and newborn. In a study among religious groups in the United States, Kaunitz and colleagues [35] demonstrated that antenatal and intrapartum care has a major impact on pregnancy outcome. Women who did not seek ANC and who delivered at home without skilled attendants had perinatal mortality three times higher, and maternal mortality 100 times higher than those who did. Observational studies, [36] also tend to show that women who receive antenatal care have lower maternal and perinatal mortality and better pregnancy outcomes. These studies also inclined to show an association between the number of antenatal visits and pregnancy outcomes. However, in recent years, apart from frequency of ANC visits and interval between the visits attention has been directed to the essential elements of ANC package, so that quality is not neglected in favour of quantity. It has thus been argued that probably, more effective care could be provided with fewer but "goal oriented" visits, especially focused on the elements of ANC that have been proven to be effective and have an impact on substantive outcome [36].

Prematurity is an important public health problem. It is the greatest cause of morbidity and mortality in obstetrics [37]. However, circumstances leading to preterm 
birth are still unclear, but its aetiology is believed to be multi-factorial [38]. Premature increase in corticotrophin-releasing hormone $(\mathrm{CRH})$ by the placenta has been linked to increased PTB rate. Also, the increased low birth weight and preterm birth in primip gravid mothers may reflect the general observation that birth weight increases with subsequent births [19].

\section{CONCLUSIONS}

The frequency of LBW and PTB in the present study is high and, was almost doubled compared to reported rates in high resource settings of the world. Pregnancies complicated by antepartum haemorrhage and hypertensive pregnancy disorders were associated with increased risk of both PTB and LBW. The implication is that newborn infants of mothers with antepartum haemorrhage and hypertensive pregnancy disorders constitute a high risk category. Thus, improvement in monitoring, timely diagnosis and management of obstetric complications through skilled attendant and timely access to EmOC services is warranted. Focused antenatal care offered as a package of interventions could substantially reduce the incidence and complications related to LBW and PTB, and subsequently increase the likelihood of newborn survival in low resource settings, such as the Gambia.

\section{ACKNOWLEDGEMENTS}

This project was funded by the Institute of General Practice and Community Medicine, University of Oslo, Norway and the Research Council. Our sincere thanks goes to the Chief Executive Officers (CEOs)/Principal Nursing officers of Bansang and Armed Forces Provisional Ruling Council (AFPRC) Hospital, and the Regional Director; Regional Health Team-NBD/W for providing office space and support during the data collection period. We also deeply appreciate the cooperation received from the data managers, nurses and midwives in the two hospitals. We are deeply indebted to our research assistants and driver for making the data abstraction possible.

\section{REFERENCES}

[1] McCormick, M.C. (1985) The contribution of lowbirth weight to infant mortality and childhood morbidity: Review. New England Journal of Medicine, 312, 82-90. doi:10.1056/NEJM198501103120204

[2] Conde-Agudelo, A., Belizan, J.M. and Diaz-Rossello, J.L. (2000) Epidemiology of fetal death in Latin America. Acta Obstetrics and Gynecology Scand, 79, 371-378.

[3] Badshah, S., Mason, L., Mckelvie, K., Payne, R. and Lisboa, P.J.G. (2008) Risk factors for low birth weight in the publich hospitals at Peshawar, NWFP-Pakistan. BMC Public Health, 8.

[4] WHO, UNICEF (2004) Low birth weight, country, regional and global estimates. WHO, Dept. of Reprod- uctive Health Research, ISBN: 92-806-3832-7, New York, 1-32.

[5] UNICEF. (2006) The State of the World's Children 2007. The United Nations Children's Fund, UNICEF, New York, 2006.

[6] The Gambia Bureau of Statistics. (2007) The Gambia multiple Cluster Indicator Survey report 2005/2006 Banjul, UNICEF, World Bank.

[7] Kramer, M.S. (1987) Determinants of low birth weight: Methodological assessment and metaanalysis. Bull World Health Organ, 65, 663-737.

[8] Steketee, R.W., Wirima, J.J., Slutsker, L., Heymann, D.L. and Breman, J.G. (1996) Problems of malaria and malaria control in pregnancy in sub-Saharan Africa. American Journal Tropical Medicine and Hygiene, 55, 2-7.

[9] McGregor, I.A. (1984) Epidemiology, malaria and pregnancy. American Journal Tropical Medicine and Hygiene, 33, 517-525.

[10] Muylder, D.X. (1989) Perinatal mortality audit in Zimbabwean district. Paediatric and Perinatal Epidem- iology, 3, 284-293. doi:10.1111/j.1365-3016.1989.tb00380.x

[11] de Onis, M., Blossner, M. and Villar, J. (1982) Level and patterns of intrauterine growth retardation in developing countries. European Journal of Clinicial Nutrition, 52 (supl 1), S5-S15.

[12] Feresu, S.A., Harlow, S.D., Welch, K. and Gillespie, B.W. (2004) Incidence of and socio-demographic risk factors for stillbirth, preterm birth and low birth weight among Zimbabwean women. Paediatr Perinat Epidemiol, 18, 154-163. doi:10.1111/j.1365-3016.2003.00539.x

[13] WHO. (1997) Coverage of Maternity Care: A listing of available information. WHO/RHT/MSM/96.28; Maternal and Newborn Health/Safe Motherhood, WHO. Bull World Health Organ, Geneva.

[14] WHO. (2004) International statistical classification of diseases and related health problems, 10th revision, 2nd Editon. Switzerland. Bull World Health Organ, Geneva.

[15] Gambia Government (2005). The standard drug treatment manual, Department of State for Health \& Social Welfare, Banjul.

[16] Rayco-Solon, P., Fulford, A.J., Prentice and A.M. (2005) Differential effects of seasonality on preterm birth intrauterine growth restrictio in rural Africans. American Journal of Clinical Nutrition, 81, 134-139.

[17] Kunzel, W., Herrero, J., Onwuhafua, P., Staub, T. and Hornung, C. (1996) Maternal and perinatal health in Mali, Togo and Nigeria. European Journal of Obstetrics and Gynecology Reproductive Biology, 69, 11-17. doi:10.1016/0301-2115(95)02528-6

[18] Ferraz, E.M., Gray, R.H. and Cunha, T.M. (1990) Determinants of preterm delivery and intrauterine growth retardation in north-east Brazil. International Journal of Epidemiology, 19, 101-108. doi:10.1093/ije/19.1.101

[19] Mavalankar, D.V., Gray, R.H. and Trivedi, C.R. (1992) Risk factors for preterm and term lowborth weight in Ahmeddaba, India. International Journal of Epidemiology, 21, 263-272. doi:10.1093/ije/21.2.263

[20] Andersson, R. and Bergström, S. (1995) Use of fundal height as a proxy for length of gestational age in rural Africa. The American Journal of Tropical Medicine Hygiene, 98, 169-172. 
[21] Engmann, C., Matendo, R., Kinoshita, R., Ditekemena, J., Moore, J. and Goldenberg, R.L., et al. (2009) Stillbirth and early neonatal mortality in rural Central Africa. International Journal of Gynaecology and Obstetrics, 105, 112-117. doi:10.1016/j.ijgo.2008.12.012

[22] Siza, J.E. (2008) Risk factors associated with low birth weight of neonates among pregnant women attending a referral hospital in Northern Tanzania. Tanzania Journal of Health Research, 10, 1-8.

[23] Sue, R. and Karen, T. (2000). Tipping the scales: Weighing in on solutions to the low birth weight problem in Colorado, Colorado Department of Public Health and Environment, 1-31.

[24] Chumnijarakij, T., Nuchaprayoon, T., Chitinand, S., Onthuam, Y. and Quamkul, N., et al. (2010) Maternal risk factors for low birth weight newborn in Thailand. Journal of Medicine Association of Thailand, 75, 445-452.

[25] Awoleke, J.O. (2011) Maternal risk factors for low birth weight babies in Lagos, ArchIves of Gynecology and Obstetrics, 6. doi:10.1007/s00404-011-1885-y

[26] Nisell, H., Palm, K. and Wolff, K. (2000) Prediction of maternal and fetal complications in preeclampsia. Acta Obstetrics and Gynecology Scand, 79, 19-23. doi:10.1080/j.1600-0412.2000.079001019.x

[27] Grujic, I. and Milasinovic, L. (2006) Hypertension, preeclampsia and eclampsia-monitoring and outcome of pregnancy. Medicinski Pregled, 59, 556-559. doi:10.2298/MPNS0612556G

[28] Duley, L. (2003) Pre.eclampsia and the hypertensive disorders of pregnancy. British Medicine Bulletin, 67, 161176. doi: $10.1093 / \mathrm{bmb} / \operatorname{ldg} 005$

[29] National High Blood Pressure Education Programme Working Group, (2000) Report of the national high blood pressure education programme working group on high blood pressure in pregnancy. American Journal of Obstetrics and Gynecology, 183, S1-S22.

[30] Roudbari, M., Yaghmaer, M. and Soheili, M. (2007) Prevalence and risk factors for low birth weight infants in
Zahedan, Islamic Republic of Iran. Eastern Mediterranean Region Health Journal, 13, 838-845.

[31] Yücesoy, G., Ozkan, S., Bordur, H., Tan, T., Caliskan, E., Vural, B. and Corakci, A. (2005) Maternal and perinatal outcome in pregnancies complicated with hypertensive disorder of pregnancy: A seven year experience of a tertiary centre. ArchIves of Gynecology and Obstetrics, 273, 43-49. doi:10.1007/s00404-005-0741-3

[32] Kato, N. and Matsuda, T. (2006) Estimation of optimal birth weights and gestational age for twins births in Japan. BMC Public Health, 6.

[33] Osungbade, K.O. and Ige, K.O. (2011) Public health perspectives of preeclampsia in developing countries: Implication for health systems strengthening. Journal of Pregnancy. doi:10:1155/2011/181095

[34] WHO, UNICEF (2003) Antenatal care in developing countries: Promises, achievements and missed opportunities An analysis of trends, levels and differentials. 1990-2001. WHO Geneva. Bulletin of World Health Organization.

[35] Kaunitz, A.M., Spence, C., Daneilson, T.S., Rochat, W.A. and Grimes, D.A. (1984) Perinatal and maternal mortality in a religious group avoiding obstetric care. American Journal of Obstetrics and Gynecology, 150, 826-831.

[36] Dowswell, T., Carroli, D., Duley, L. and Gates, S., et al. (2010) Alternative versus standard package of antenatal care for low-risk pregnancy (review). The Cochrane Database of Systematic Review, 10. doi:10.1002/14651858.CD00934.pub2

[37] Reedy, N.J. (2007) Born too soon. The continuing challenges of preterm labour and birth in the United States. Journal of Midwifery \& Women's Health, 52, 281-290. doi:10.1016/j.jmwh.2007.02.022

[38] Beck, S., Wojdyla, D., Say, L., Betran, A.P., Merialdi, M. and Requejo, J.H., et al. (2010) The worldwide incidence of preterm birth: A systemic review of maternal mortality and morbidity. Bulletin of World Health Organization, 88, 31-38. doi:10.2471/BLT.08.062554 